\title{
Transposons and Tandem Repeats Are Not Involved in the Control of Genomic Imprinting at the MEDEA Locus in Arabidopsis
}

\author{
C. Spillane, ${ }^{* \dagger}$ C. Baroux, ${ }^{*}$ J.-M. Escobar-Restrepo, ${ }^{*}$ D.R. Page, ${ }^{*}$ \\ S. LAOUEILle, ${ }^{\dagger}$ and U. GrossnikLAUS* \\ "Institute of Plant Biology and Zürich-Basel Plant Science Center, University of Zürich, 8008 Zürich, \\ Switzerland; 'Plant Molecular Genetics, Department of Biochemistry, University College Cork, Cork, Ireland
}

\begin{abstract}
Transposons are selfish mobile DNA elements that can insert into nonhomologous target sites, thereby amplifying their copy number in the genome. Yet, transposition is considered tightly controlled, because unregulated amplification could have severe consequences for the fitness of the host organism. Nonetheless, transposons constitute $\sim 45 \%$ of the human genome and $\sim 80 \%$ of the maize genome. Transposable elements can act as both "attractors" and "mediators" of epigenetic regulation across the genome. The potential for transposons to show epigenetic activity leading to effects on phenotypic variation was first recognized by McClintock (see McClintock 1984) as "changes in phase." Some years ago it was proposed that transposons may be involved in a variety of epigenetic phenomena such as gene silencing, paramutation, and genomic imprinting (Martienssen 1996; Matzke et al. 1996). Indeed, there is growing evidence that transposons can act as epigenetic mediators of phenotypic variation. Here, we briefly review the role of transposons and repeated sequences in epigenetic gene regulation and investigate their potential role in controlling genomic imprinting at the MEDEA locus of Arabidopsis.
\end{abstract}

\section{TRANSPOSONS AND REPETITIVE SEQUENCES AS EPIGENETIC ATTRACTORS AND MEDIATORS}

Transposons are well known to be evolutionary drivers of chromosomal repatterning by reorganizing genome structure through transposition and by causing chromosomal rearrangements such as deletions, inversions, and translocations. But they can also modulate the transcription patterns of genes adjacent to the sites of transposon insertions (for review, see Kazazian 2004). Over evolutionary time most transposons have accumulated mutations that render them incapable of transposition, but many of their promoters remain active (Whitelaw and Martin 2001). Retrotransposons often have strong constitutive promoters that can affect the transcription of adjacent genes. Indeed, transposons can serve as alternative promoters for many mammalian genes (van de Lagemaat et al. 2003). For instance, transposon insertions proximal to genes can lead to overexpression causing hypermorphic alleles or to the production of chimeric transcripts that encode proteins with anti- or neomorphic activity. Such effects have been demonstrated for the agouti (Michaud et al. 1994; Argeson et al. 1996) and the mouse intracysternal A-type particle (IAP)-promoted Mipp gene (Chang-Yeh et al. 1993).

The effects described above may be viewed as a consequence of genetic changes since they rely on a readthrough transcription from a transposon promoter and thus a restructured genome. However, transposons may also provide a link between genetic and epigenetic processes, via their activities as both transcribed genes and cis-acting repeats. In many organisms, duplicated or repetitive elements including transposons act as epigenetic "attractors" of mechanisms that lead to their inactivation or reduce their copy number (Matzke et al. 1996; Yoder et al. 1997; Whitelaw and Martin 2001; Lyon 2003). The mechanisms differ between repeat systems and organisms, but involve many of the classical epigenetic regulatory systems, such as DNA methylation, chromatin modification, and transcriptional interference.

\section{DNA Methylation and Histone Modifications Regulate Transposon Activity}

It has been proposed that cytosine DNA methylation acts primarily to suppress transcription from "intragenomic" parasitic elements (e.g., transposons) across the genomes of higher eukaryotes (Matzke et al. 1996; Yoder et al. 1997; Bestor 2003). DNA methylation can suppress transposition by making the transposon inaccessible to its transposase. In addition, transposon promoters can be inactivated by methylation either epigenetically or genetically, because of the increased frequency of $\mathrm{C} \rightarrow \mathrm{T}$ base transitions at methylated sites. Studies in both mammals and plants have demonstrated that demethylation of the genome can trigger remobilization of epigenetically silenced transposons (Walsh et al. 1998; Hirochika et al. 2000; Kato et al. 2003). It is likely that the majority of cytosine methylation found in eukaryotic genomes is associated with suppression of multicopy transposons and centromeric satellite DNA (both enriched for $\mathrm{CpG}$ content). This seems to be the case for plants and fungi where methylation is mainly associated with transposons and repetitive DNA, whereas in mammals coding regions also 
can be methylated (Martienssen and Colot 2001; Lippman et al. 2004). Transposon insertions may also affect adjacent genes via spreading of $\mathrm{CpG}$ methylation into "innocent bystander" genes, leading to their silencing. Yates and colleagues (1999) demonstrated such an effect for tandem $\mathrm{B} 1$ repetitive elements on the silencing of the adjacent adenine phosphoribosyltransferase Aprt gene in mice.

In recent years it has become clear that histone modifications play an important role in the epigenetic regulation of gene activity (for review, see Imhof 2003; Wang et al. 2004). There are mechanistic links between DNA methylation and histone modifications affecting chromatin structure (for review, see Tariq and Paszkowski 2004), and transposons can also be suppressed by targeting them for heterochromatin formation, which would act to suppress their transcription, mobility, and recombinational activity. Indeed, studies of transposons have demonstrated that transposon loci are subject to histone methylation (Rea et al. 2000; Gendrel et al. 2002). For instance, Arabidopsis mutants affecting epigenetic regulation were investigated for effects on the activity and inheritance of six transposon classes (Lippman et al. 2003). It was found that two distinct epigenetic mechanisms silence transposons and that transposon silencing complexes interact via histone modifications and RNA interference (RNAi). There is mounting evidence for a role for RNAi in chromatin modifications that regulate transposable element activity at centromeric heterochromatin (Volpe et al. 2002; Dawe 2003). In Caenorhabditis elegans, RNAi-deficient strains exhibit mobilization of endogenous transposons indicating that the RNAi machinery is involved in suppression of transposon activity (Tabara et al. 1999). The emerging picture is that heterochromatic regions can generate small RNAs that direct an RNAi-based modification of the chromatin in heterochromatic repeats and transposable elements (Bender 2004; Lippman and Martienssen 2004).

\section{Transposons and Classical Epigenetic Phenomena}

Metastable epialleles are alleles where the epigenetic state can switch and be mitotically inherited, yet the establishment of the epigenetic state is a probabilistic event (Rakyan et al. 2002). All metastable alleles that have been investigated at the molecular level have been shown to be associated with a transposon insertion (Rakyan et al. 2002). For instance, Rakyan and coworkers (2003) have demonstrated a role for retrotransposon-based regulation of the classical metastable mutant allele Axin-fused $\left(A x i^{F u}\right)$. The presence or absence of the Axin ${ }^{F u}$ phenotype, a kinked tail, correlated with differential DNA methylation of a retrotransposon within $\mathrm{Axin}^{\mathrm{Fu}}$. Affected transcripts arising adjacent to the retrotransposon long terminal repeat (LTR), usually containing a promoter, are considered as likely causes of the phenotype. A similar case was described for an agouti allele, where the insertion of an IAP retrotransposon into the upstream region caused a range of phenotypes that showed partial epigenetic maternal inheritance due to incomplete erasure of the epigenetic modification at agouti (Morgan et al. 1999).
Transposons are also proposed to play a role in mammalian X-chromosome inactivation. Interspersed repeats, in particular long interspersed nucleotide elements (LINEs), have been suggested as features that act as attractors of the X-inactivation machinery (Lyon 2003). In support of this model is the observation that L1 LINE content is lower in regions of the $\mathrm{X}$ chromosome containing genes that escape inactivation (Bailey et al. 2000).

Several years ago it was proposed that transposons and repetitive elements may be mechanistically linked to the phenomenon of paramutation (Martienssen 1996; Matzke et al. 1996; Della Vedova and Cone 2004). Paramutation is an allelic interaction that results in meiotically heritable changes in gene expression (Brink 1973). By analyzing the physical structure of 28 haplotypes at the red $1(r 1)$ locus of maize, a strict correlation of paramutability (the ability to become silenced) and structural features could be established (Walker and Panavas 2001). The $r l$ locus is complex, often containing several $r l$ gene copies encoding helix-loop-helix transcription factors. All paramutable alleles contain an $S$ subcomplex that includes two $S$ genes ( $r 1$ homologs) forming a head-to-head inverted repeat and a $q$ gene fragment (homologous to the $r l$ promoter). These elements of the $S$ subcomplex usually contain sequences derived from a Doppia transposable element. The paramutagenicity (the ability to cause silencing) of $r l$ haplotypes, on the other hand, does not correlate with structural features but paramutagenic alleles show consistently higher levels of DNA methylation (Walker and Panavas 2001). At the boosterl (b1) locus, which is also subject to paramutation, Stam and coworkers (2002) have shown that tandem repeats of an 853-bp sequence located $\sim 100 \mathrm{~kb}$ upstream of the $b 1$ gene are required for paramutagenicity. A further link between paramutation and transposons is illustrated by the fact that paramutation at three different loci in maize and silencing of Mutator transposable elements are coordinately affected in certain inbred backgrounds (Walbot 2001) and by mutations at the modifier of paramutation 1 (mop 1) locus (Dorweiler et al. 2000; Lisch et al. 2002).

\section{EPIGENETIC REGULATION OF TRANSPOSONS AND IMPRINTED GENES}

Genomic imprinting refers to an epigenetic phenomenon where paternally and maternally inherited alleles are expressed differentially after fertilization. Most imprinted genes in mammals display parent-of-originspecific methylation patterns. Compelling evidence that transposons are not neutral genomic parasites but actively influence epigenetic gene regulation poses the question whether they play a role in genomic imprinting as well. Indeed, the epigenetic regulation of some transposons is analogous to that of imprinted genes whereby an autosomal locus can be differentially expressed depending on the sex of the parent from which it was inherited. In mammals, L1 elements and IAP retroviruses are methylated when inherited paternally, but not methylated when inherited maternally (Sanford et al. 1987). The opposite situation occurs for mammalian Alu elements (Rubin et al. 
1994). Early during mouse embryogenesis, genome-wide DNA demethylation occurs, followed by de novo remethylation. For most imprinted genes, the unmethylated allele escapes postimplantation de novo methylation; differentially methylated transposons, however, do not (Yoder et al. 1997; Walsh et al. 1998).

A survey of more than 30 imprinted genes brought the first correlative evidence for a possible involvement of transposons in genomic imprinting. Neumann et al. (1995) highlighted that one of the characteristics of known imprinted genes was that they tended to be enriched in short direct repeats. In mammals, the accumulation of short interspersed nucleotide elements (SINEs) is constrained in promoter regions of imprinted genes, whereas L1 LINE transposons preferentially accumulate in the vicinity of paternally expressed imprinted genes (Greally 2002; Fazzari and Greally 2004). Furthermore, this dual feature of imprinted regions points toward a mechanistic role of the transposons, where paucity in one type (the SINEs) would ensure that imprinted regions are isolated in a distinct genomic compartment, potentially enabling distinct regulatory mechanisms, and the other type (the L1 LINEs), being asymmetrically distributed, would provide a genomic signature to undergo preferential maternal or paternal silencing in the gametes. This remains a postulate, which needs to be experimentally verified, but it opens the field of investigation toward elucidating the mechanisms of genomic imprinting.

\section{GENOMIC IMPRINTING IN PLANTS}

Long before genomic imprinting was studied in mammals, Kermicle (1970) demonstrated that specific alleles of the maize $r 1$ locus are regulated by genomic imprinting (for review, see Kermicle 1994; Baroux et al. 2002). Maternal and paternal alleles of several other maize and, more recently, Arabidopsis genes were shown to be differentially expressed during seed development. However, of all plant genes suggested to be regulated by genomic imprinting, the maternally inherited allele is active, and most of these genes are already expressed prior to fertilization. Thus, although unlikely, the differential steadystate levels of maternally and paternally derived transcripts might be due to expression of the maternal allele prior to fertilization and not due to active expression postfertilization. In such cases, a clear demonstration of genomic imprinting requires not only the detection of differential expression levels of maternally and paternally derived transcripts but also an assay showing that the maternal allele is actively transcribed in at least one of the products of double fertilization (embryo and endosperm).

Regulation by genomic imprinting was proposed for several maize genes that are active in the endosperm, including specific alleles of $r 1$ (Kermicle 1970), the delta zein regulatorl gene ( $d z r 1$; Chaudhuri and Messing 1994), and specific $\alpha$-tubulin and zein genes (Lund et al. 1995a,b). Except for $d z r 1$, which is not yet cloned, the high expression levels of maternal genes correlated with hypomethylation of the maternally inherited alleles (for review, see Alleman and Doctor 2000; Baroux et al.
2002). For $r l$, genomic imprinting could unambiguously be demonstrated in an elegant genetic analysis that excluded a prefertilization component (for instance, longlived, stored transcripts [Kermicle 1970]). For the other potentially imprinted loci, early expression in ovules was not analyzed; therefore, a prefertilization cause for differential transcript levels cannot unambiguously be excluded. Recently, three additional endosperm-specific maize genes have been described that show differential expression levels during seed development but are not expressed at all prior to fertilization. Thus, ZmFiel (Springer et al. 2002; Danilevskaya et al. 2003), no apical meristem related protein1 ( $n r p 1$; Guo et al. 2003), and maternally expressed genel (meg1; Gutiérrez-Marcos et al. 2004) are clearly regulated by genomic imprinting.

\section{The FIS-Class Genes in Arabidopsis}

In Arabidopsis, the MEDEA (MEA) gene, which was isolated in a screen for gametophytic maternal effect mutations (Grossniklaus et al. 1998), was shown to be regulated by genomic imprinting. Differential expression levels of transcripts derived from the two parental alleles were demonstrated by allele-specific reverse transcriptase polymerase chain reaction (RT-PCR) (Kinoshita et al. 1999; Vielle-Calzada et al. 1999) and active transcription after fertilization was shown using an in situ hybridization method analogous to RNA-FISH (fluorescence in situ hybridization) (Vielle-Calzada et al. 1999). This analysis showed that only two of the three $M E A$ copies present in the endosperm are actively transcribed. Although the latter method could be applied only to endosperm nuclei, allele-specific quantitative PCR analyses showed that paternally derived transcripts were not detectable at any stage of seed development (up to 10 days after pollination; Page 2004). Thus, the $M E A$ gene, which is expressed in both embryo and endosperm (VielleCalzada et al. 1999), is likely regulated by genomic imprinting in both fertilization products.

$M E A$ encodes a Polycomb group (PcG) protein with high similarity to Enhancer of zeste from Drosophila (Grossniklaus et al. 1998). Several other independent screens identified additional loci with similar parent-oforigin-dependent phenotypes (Ohad et al. 1996; Chaudhury et al. 1997; Guitton et al. 2004). This class of mutations is referred to as the fis class (Grossniklaus et al. 2001) and includes the MEA (Grossniklaus et al. 1998), FERTILIZATION-INDEPENDENT ENDOSPERM (FIE; Ohad et al. 1999), FERTILIZATION-INDEPENDENT SEED2 (FIS2; Luo et al. 1999), and MSI1 (Köhler et al. 2003 ) genes. These FIS proteins form a multiprotein complex that is analogous to the $\mathrm{E}(\mathrm{z})$-Esc complex of Drosophila and the Enx-Eed complex of mammals (Köhler et al. 2003; for review, see Reyes and Grossniklaus 2003). Differential expression of maternally and paternally inherited alleles has been described for the FIE and FIS2 genes (Luo et al. 2000; Yadegari et al. 2000). However, since both of these genes are also expressed prior to fertilization (Luo et al. 2000: Spillane et al. 2000; Yadegari et al. 2000) and active transcription at postfertilization 
stages has not been investigated, regulation by genomic imprinting has not been demonstrated unambiguously.

\section{Transposons and Genomic Imprinting at the FWA Locus}

Recently, genomic imprinting was reported for an additional Arabidopsis gene: the FWA locus (Kinoshita et al. 2004), which was originally identified as a late flowering mutant (Koornneef et al. 1991). Late flowering in the fwa epimutant is caused by ectopic expression of the $F W A$ gene due to hypomethylation of repeats upstream of the transcriptional start site (Soppe et al. 2000). Kinoshita et al. (2004) found that $F W A$ is expressed in the endosperm and could detect transcripts derived only from the maternally inherited allele, suggesting the gene may be regulated by genomic imprinting. However, as $F W A$ is also expressed prior to fertilization and a conclusive test for active transcription at postfertilization stages has not been performed, the definitive proof is missing. Nevertheless, it is highly likely that $F W A$ is regulated by genomic imprinting because it also shares upstream regulators with $M E A$. On the one hand, the maternal activity of both $M E A$ and FWA depends on DEMETER (DME), a gene that was identified based on its phenotype that is similar to that of mea (Choi et al. 2002; Guitton et al. 2004). DME encodes a DNA-glycosylase homolog whose activity is required for an active maternal $M E A$ allele (Choi et al. 2002, 2004). On the other hand, the DNA methyltransferase MET1 is a regulator of both genes. However, while MET1 was proposed to act antagonistically to $D M E$ on the maternal $M E A$ allele (Xiao et al. 2003), it repressed the paternal $F W A$ allele (Kinoshita et al. 2004), while $M E T 1$ does not seem to affect the paternal $M E A$ allele (Luo et al. 2000).

Interestingly, at the Symposium, Robert Martienssen (see Martienssen et al., this volume) reported a link between the imprinted control of $F W A$ expression and transposons. His group investigated McClintock's hypothesis (1952) that transposons ("controlling elements") might reside in heterochromatic regions (for instance, heterochromatic knobs), but also exercise regulatory functions across the genome. In support of this hypothesis, Lippman and coworkers (2004) have demonstrated that heterochromatin in Arabidopsis is determined by transposons and related tandem repeats, which are epigenetically regulated by the chromatin remodeling ATPase DDM1. It was further shown that transposons can exercise epigenetic regulation of adjacent genes and that this was the likely explanation for the epigenetic inheritance patterns observed at the imprinted $F W A$ gene. In addition, small interfering RNAs (siRNAs) associated with the epigenetically regulated transposon type were found in both heterochromatin and the promoter of the $F W A$ locus. It was proposed that the transposon brings the $F W A$ locus under the control of $D D M 1$ and is responsible for its epigenetic regulation (Lippman et al. 2004).

Over the last few years, we have investigated the potential role of transposable elements and repeated sequences in the regulation of the imprinted $M E A$ locus. Unlike for
$F W A$, no evidence for a role of repeats was found, suggesting that different mechanisms are responsible for the regulation of $M E A$ and $F W A$ by genomic imprinting.

\section{EXPERIMENTAL PROCEDURES}

\section{Plant Material and Growth Conditions}

The mea- 1 mutant line in Ler-0 genetic background used was previously described (Grossniklaus et al. 1998). All Arabidopsis ecotypes were obtained from the Arabidopsis Biological Resource Center (ABRC) at Ohio State University. Seeds were surface sterilized using 2\% sodium hypochlorite and allowed to germinate on Murashige and Skoog (MS) medium (Duchefa) supplemented with $10 \mathrm{~g} / 1$ of sucrose, $8 \mathrm{~g} / 1$ of agar prior to transfer of seedlings to soil. To generate interecotype F1 hybrids between the mea-1 mutant and the ecotypes Ler-0, Yo- 0 , and $\mathrm{Kb}-0$, each ecotype was crossed with pollen from a mea-1 plant and the hybrid F1 progeny seeds were selected on MS medium containing $50 \mathrm{mg} / \mathrm{L}$ of kanamycin (Sigma) as described above. Interecotype F1 mea-1 seedlings displaying kanamycin resistance were chosen for further analysis. Seedlings of both ecotypes and F1 interecotype progeny were transplanted to "ED73 mit Bims" soil (Tränkle Einheiteserde) and transferred to a growth chamber with $70 \%$ humidity and a day/night cycle of 16 hours light at $21^{\circ} \mathrm{C}$ and 8 hours dark at $18^{\circ} \mathrm{C}$.

A set of 50 evolutionarily divergent ecotypes (comprising a core collection kindly provided by Tom MitchellOlds, MPI Jena) was chosen for genetic and molecular analyses. These ecotypes were (stock center accession numbers in parentheses) Ler-0 (CS20), Mh-0 (CS904), Blh-1 (CS1030), Cit-0 (CS1080), Co-1 (CS1084), Col-0 (CS1092), Cvi-0 (CS1096), Di-0 (CS1106), Ei-2 (CS1124), Est-0 (CS1148), Fe-1 (CS1154), Ga-0 (CS1180), Gr-1 (CS1198), Gu-0 (CS1212), Ha-0 (CS1218), Ita-0 (CS1244), Kas-1 (CS1264), Kb-0 (CS1268), Kil-0 (CS1270), Kin-0 (CS1272), Le-0 (CS1308), L1-0 (CS1338), Lo-1 (CS1346), Lz-0 (CS1354), Me-0 (CS1364), Nd-0 (CS1390), Np-0 (CS1396), Nok-0 (CS1398), Pa-1 (CS1438), Pla-0 (CS1458), Pog-0 (CS1476), Rsch-0 (CS1490), Ru-0 (CS1496), Sah-0 (CS1500), Ta-0 (CS1548), Tu-1 (CS1568), Uk-1 (CS1574), Ws-0 (CS1602), Wt-1 (CS1604), Yo-0 (CS1622), WI-0 (CS1630), Wei-0 (CS3110), RLD1 (CS913), XX-0 (N1618), Mt-0 (N1380), Ko-2 (N1288), C24 (N906), CS22491, CS22495, CS22484, CS22493, and Hodja.

\section{Molecular Biology}

Genomic DNA template for PCR and sequence analysis of each of the ecotypes was extracted as described (Edwards et al. 1991). To determine whether the $M E A$ AtREP2 helitron was present in each ecotype, a PCR assay was developed using two primers spanning the $M E A$ AtREP 2 insertion site. The primers used were MEAP RAD S1: 5'-GATATGTTGG GTCCGTCGG-3' and MEAP RAD AS1: 5'-CTATGCT CGTCTAGCTAC-3'. 
For the PCR analysis spanning the MEA-AtREP2 helitron region, the $\mathrm{PCR}$ conditions consisted of annealing temperature of $55^{\circ} \mathrm{C}(15 \mathrm{sec})$ and extension time of $30 \mathrm{sec}-$ onds for 30 cycles.

A series of four different combinations of primer pairs were used for PCR spanning the MEA-ISR region. Primer pair 1 consisted of MEA S40: $5^{\prime}$-GCTATGGACC AGAACATGC-3' and MEA AS42: 5'-AGGGTTTGCT CTTGAAGTCAG-3'. Primer pair 2 consisted of MEA3 REP1: 5'-GTGGCTGTAGCTTACGAAAGG-3' and MEA AS42: 5'-AGGGTTTGCTCTTGAAGTCAG-3'. Primer pair 3 consisted of MEA 3'REP1F: 5'-GTGGCT GTAGCTTACGAAAGG-3' and MEA 3'REP2R: 5' GTTTGGATTCGTGATATACACC-3'. Primer pair 4 consisted of MEA S40: 5'-GCTATGGACCAGAACATGC-3' and MEA3'REP2R: 5'-GTTTGGATTCGTG ATATACACC-3'. For the PCR analysis spanning the MEA-ISR, the PCR conditions were annealing temperature of $50^{\circ} \mathrm{C}(15 \mathrm{sec})$ and extension time of 90 seconds for 30 cycles. BamHI restriction analysis of PCR products was conducted using standard protocols on MEA-ISR PCR products obtained from the following ecotypes: Bla1, Bla-14, Blh-1, C24, Cit-0, Co-1, Co-2, Col-0, Col-1, Cs22493, Cs22495, Ct-1, Cvi-0, Di-0, Ei-2, Est-0, Estland, Fr-2, Ga-0, Gr-1, Gu-0, Ha-0, Hodja, Kas-1, Kb-0, Kil-0, Kin-0, KN-0, Le-0, Ler-0, Lo-1, Me-0, Ms-0, Mt0, Nd-0, No-0, Nok-0, Np-0, Pa-1, Pog-0, RLD1, Rsch-0, Ru-0, Sah-0, Sf-1, Te-0, Tsu-0, Tu-1, UK-1, Wl-0, Wil1 , Ws, Ws-0, Wt-1, XX-0, and Yo-0. All of these ecotypes produced a PCR product with at least one primer pair combination spanning the MEA-ISR.

\section{Bioinformatics}

The MEA-AtREP2 helitron element was initially identified using the repeat element mapping program Repeat View (http://www.itb.cnr.it/webgene/). The CpG islands were identifed using the $\mathrm{CpG}$ islands prediction program available on the Webgene Web site (http://www. itb.cnr.it/webgene/). The $M E A$ and $F W A$ tandem repeats were identified by the Tandem Repeats Finder program (http://c3.biomath.mssm.edu/trf.basic.submit.html). The large tandem duplication in the $M E A$ upstream region was identified from restriction enzyme profiles of the $M E A$ locus that exhibited similar restriction patterns indicative of a tandem duplication.

\section{RESULTS AND DISCUSSION}

\section{Transposons and Tandem Repeats at the $M E A$ and $F \boldsymbol{W A}$ Loci}

To determine whether the two imprinted loci $M E A$ and FWA in Arabidopsis contained any common structural features that could be associated with epigenetic regulation (i.e., genomic imprinting) we used a range of bioinformatics tools for comparative purposes. The sequences analyzed were generated by the Arabidopsis Genome Initiative (2000) and represent the Colombia (Col-0) accession.

In mammals, the regulatory regions of imprinted genes frequently contain a combination of features including tandem repeats associated with differentially methylated CpG islands (Moore 2001). Transposable elements are found in the upstream regions of both $F W A$ and $M E A$, although each imprinted gene is proximal to a different type of transposable element in their upstream regions. While FWA contains an AtSINE3 element 980 bp upstream of its start codon, the closest transposable element to the imprinted $M E A$ locus is an AtREP2 helitron found $4363 \mathrm{bp}$ upstream of the $M E A$ start codon (Fig. 1A).

The imprinted $M E A$ and $F W A$ loci also contain tandem repeats (Fig. 1). The $M E A$ upstream region contains two tandemly duplicated segments $(\sim 1450 \mathrm{bp}$ and $\sim 1690 \mathrm{bp})$ spanning $\sim 3140 \mathrm{bp}$. However, no analogous large tandem
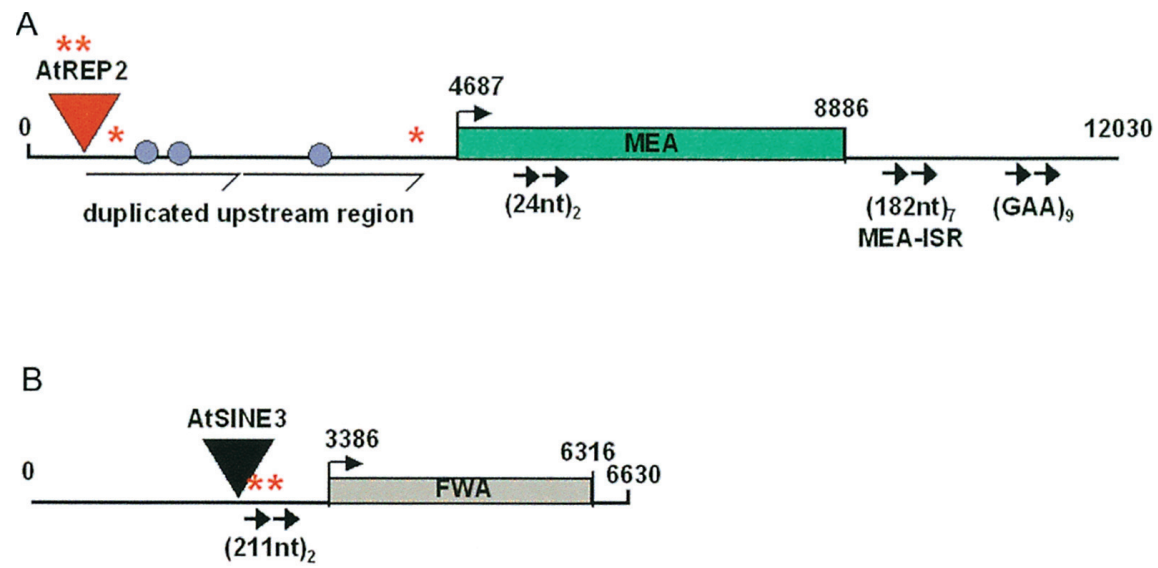

Figure 1. cis-elements in known imprinted Arabidopsis genes as candidate imprinting control elements (ICEs). (A) MEA locus (Col0 accession) with AtREP2 helitron transposon (triangle) and $\mathrm{CpG}$ islands (circles). Methylated regions upstream of $M E A$ as previously reported for stamen and entire seeds (Xiao et al. 2003) are represented as asterisks. MEA-ISR refers to MEA intergenic subtelomeric repeat region consisting of eight tandem repeats of 182 nucleotides in Col-0. Other repeats at the MEA locus are a tandem 24-nucleotide repeat and a trinucleotide GAA repeated nine times. (B) FWA locus (Col-0 accession) with a AtSINE3 transposon (triangle) and tandem 211-nucleotide repeat in the upstream region. Methylated regions as previously reported for embryo, seed coat, and vegetative tissues (Soppe et al. 2000; Kinoshita et al. 2004) are represented by asterisks. 
duplications are found at the imprinted $F W A$ locus. The $M E A$ locus contains three different types of tandem repeats: (i) a 24-nucleotide tandem doublet in the third exon of the $M E A$ ORF, (ii) a downstream tandem repeat region of seven 182 nucleotide repeats $(182 \mathrm{nt})_{7}$, and (iii) a downstream GAA trinucleotide (GAA) 9 . The $F W A$ locus contains less extensive tandem duplications than the $M E A$ locus and contains simply a 211-nucleotide tandem doublet 977 bp upstream of the FWA start codon. We also determined whether each of the two imprinted loci contain $\mathrm{CpG}$ islands and found that the imprinted $M E A$ locus contains $3 \mathrm{CpG}$ islands $3331 \mathrm{bp}, 3028$ bp, and 1215 bp upstream of its start codon, but the imprinted $F W A$ locus contains no $\mathrm{CpG}$ islands that we could detect using the $\mathrm{GpC}$ island prediction program.

\section{The AtREP2 Helitron Is Not Required for Imprinting at the $M E A$ Locus}

The 5' upstream region of $M E A$ contains a nonautonomous AtREP2-type Helitron transposable element (Kapitonov and Jurka 2001). Helitrons are a novel class of eukaryotic DNA transposons that can transpose by rolling circle replication (Kapitonov and Jurka 2001). Both autonomous and nonautonomous helitrons can be found in eukaryotic genomes. The autonomous rollingcircle (RC) helitrons encode a 5'-to-3' DNA helicase and nuclease/ligase similar to those encoded by known RC replicons. In addition, numerous nonautonomous RC helitron derivatives can be found throughout some eukaryotic genomes. In C. elegans, helitrons (autonomous and nonautonomous) can constitute $\sim 2 \%$ of the genome
(Kapitonov and Jurka 2001). The MEA-AtREP2 element is a nonautonomous helitron.

We investigated whether presence of the AtREP2 transposable element 4363 bp upstream of the $M E A$ start codon (Fig. 1A) in the Col-0 and Ler-0 ecotypes is correlated with genomic imprinting at the $M E A$ locus taking advantage of the natural variation resources available in Arabidopsis. It is known that transposons can accumulate to varying extents between Arabidopsis accessions. For instance, a few classes of DNA transposons have been found to be completely absent from some Arabidopsis accessions, yet they are prevalent in others. Examples include the low-frequency Tagl element absent from Colombia and WS (Frank et al. 1998) and the CACTA family transposons absent from C24 (Kato et al. 2003). However, it is not known whether the RC helitrons display any significant polymorphism between Arabidopsis ecotypes.

To identify ecotypes lacking the MEA-AtREP2 helitron insertion, we used a PCR-based strategy to amplify across the MEA-AtREP2 insertion site in 33 evolutionarily divergent Arabidopsis accessions, four of which are shown in Figure 2. Out of 33 ecotypes screened from an Arabidopsis core collection, we identified nine ecotypes where an $\sim 250$-bp PCR product was observed (see example in Fig. 2B for Yo-0 and $\mathrm{Kb}-0$ ). As this size is smaller than the expected $\sim 850 \mathrm{bp}$, these were candidates where the MEA-AtREP2 insertion may be absent. To confirm that the $A t-R E P 2$ helitron was indeed absent in these accessions, we sequenced of the PCR product spanning the $M E A-A t R E P 2$ insertion site (Fig. 2C). This analysis showed that several accessions, including Yo-0 and $\mathrm{Kb}-$

A

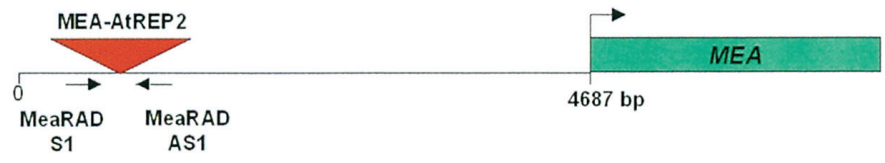

B

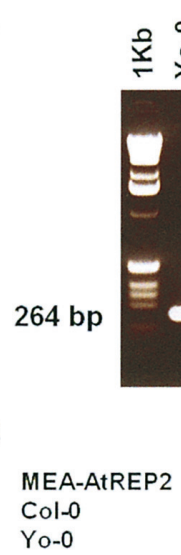

点

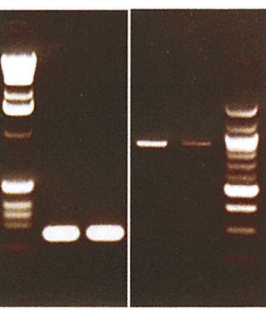

848 bp

Yo-0

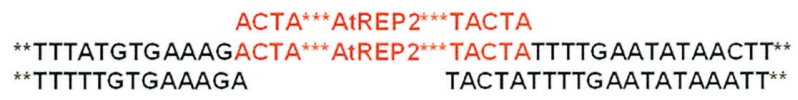

Figure 2. MEA-AtREP2 helitron is absent in accessions where $M E A$ remains imprinted. (A) Schematic representation of $M E A$ locus indicating $M E A-A t R E P 2$ helitron transposon upstream of MEA. Primers (MeaRAD S1 and Mea RAD AS1) spanning the MEAAtREP2 insertion are represented by arrows. $(B)$ Gel electrophoresis of PCR products of four different ecotypes, indicating that this $M E A$-AtREP2 is not present in the accessions $\mathrm{Yo}-0$ and $\mathrm{Kb}-0$. (C) Sequence analysis across the MEA-AtREP2 insertion site was used to confirm that the MEA-AtREP2 helitron was absent in accessions such as Yo-0. 
0, completely lack this AtREP2 helitron.

To link these data with genomic imprinting we investigated whether the accessions lacking the AtREP2 transposons still contain an imprinted $M E A$ locus. A genetic study performed in our laboratory aimed at the identification of modifiers of genomic imprinting at the $M E A$ locus within the Arabidopsis gene pool (C. Spillane and U. Grossniklaus, unpubl.). To determine whether an accession contained any cis- or trans-acting modifiers of mearelated seed abortion we crossed each accession with a mea/MEA pollen donor, and selected $\mathrm{F} 1$ hybrids with the genotype mea/MEA. Analysis of the F2 seed (aborted: normal) from the selfed mea/MEA F1 hybrids allowed us to test whether any genetic modifiers of mea-related seed abortion were present. For instance, under the assumption that paternally supplied $M E A$ activity can rescue mea maternal effect seed abortion, the survival of a seed inheriting a mutant mea allele from the mother would indicate that the paternally inherited $M E A$ allele was active unlike in the Ler accession. In general, any modifier leading to the survival of seeds that inherited a mutant maternal mea allele, or to the abortion of seeds that inherited a wildtype maternal $M E A$ allele, distort the $50 \%$ seed abortion ratio observed in heterozygous mea/MEA plants (Grossniklaus et al. 1998). Thus, if we observed a F2 seed abortion ratio of $50 \%$ from a selfed mea/MEA F1 hybrid, the accession contains no modifier, whether paternally, maternally, or zygotically acting. This reasoning applies irrespective of whether the imprint corresponds to the maternal activated state, the paternal silent state, or both. Our analysis showed that the accessions $\mathrm{Yo}-0$ and $\mathrm{Kb}-0$ do not contain any genetic modifiers of genomic imprinting, because all seeds inheriting a mutant maternal mea allele abort (Table 1). As both of the ecotypes Yo-0 and $\mathrm{Kb}-0$ lack the MEA-AtREP 2 insertion upstream of the $M E A$ locus, this strongly suggests that the MEA-AtREP2 helitron is not involved in imprinting at the $M E A$ locus.

\section{The Repetitive MEA-ISR Tandem Repeats Are Not Required for Imprinting at the MEA Locus}

Direct tandem repeats have been found proximal to several imprinted genes in mice and humans. The "tandem repeat hypothesis" has been proposed, suggesting that repeats may be important in targeting methylation to differentially methylated regions (DMRs) (Neumann et al. 1995; Lewis et al. 2004). In mammals, evidence for a causal role for tandem repeats in imprinting regulation remains inconclusive (Lewis et al. 2004). In plants, it has been proposed that tandem repeats associated with a SINE transposable element insertion and associated tandem repeats adjacent to the $F W A$ gene are the likely cause of imprinting at the FWA locus (Lippman et al. 2004).

Downstream from the $M E A$ gene we found a conspicuous cluster of short repeats. These 182 nucleotide repeats are also found in 12 other genomic locations in the Arabidopsis genome, all of which are also subtelomeric. Hence, Cao and Jacobsen (2002) named the $(182)_{7}$ repeat region MEA-ISR for intergenic subtelomeric repeat region. They showed that this region attracts high levels of DNA methylation in wild-type strains, namely, $87 \%$ at $\mathrm{CpG}, 47 \%$ at $\mathrm{CpNpG}$, and $18 \%$ at asymmetric sites. All asymmetric and $\mathrm{CpNpG}$ methylation was abolished at the MEA-ISR (and also at the FWA $211 \mathrm{nt}$ direct repeats) in $d r m 1, d r m 2$ double-mutant and drm1,drm2; cmt3-7 triple-mutant backgrounds, while $\mathrm{CpG}$ methylation levels remained similar to the wild type (Cao and Jacobsen 2002). Subsequently, Zilberman et al. (2003) demonstrated that the ARGONAUTE4 (AGO4) gene involved in RNA-mediated silencing was also necessary for asymmetric and $\mathrm{CpNpG}$, but not $\mathrm{CpG}$, methylation at the MEA-ISR. In contrast, loss of $A G O 4$ activity had no effect on asymmetric, $\mathrm{CpNpG}$, or $\mathrm{CpG}$ methylation at the FWA 211-nucleotide direct repeats.

To determine whether the MEA-ISR region is involved in genomic imprinting at the $M E A$ locus, we used a combination of PCR and restriction enzyme-based assays. We screened evolutionarily divergent accessions from an Arabidopsis core collection to determine whether any accession lacked the MEA-ISR. For the 56 ecotypes for which we obtained PCR products, our results indicate that the MEA-ISR region has undergone substantial expansions and contractions between accessions, likely because of differences in the number of the 182-nucleotide repeats in each accession (Fig. 3).

In the Col-0 genome, each of the 182-nucleotide repeat regions in the MEA-ISR contains a BamHI (B) restriction site (Fig. 3A). This allowed us to develop a simple assay to test whether each PCR product obtained from primers (e.g., MEA S40 and MEA AS42) spanning the MEA-ISR contained at least one BamHI site. We were interested to identify MEA-ISR-derived PCR products containing no Bam HI sites, as these are candidates where the 182 -nucleotide repeat region may be absent. BamHI restriction analysis of PCR products from 19 different ecotypes indicated that one or more BamHI sites were present in the MEA-ISR region in all 19 ecotypes tested (results not

Table 1. The Presence of Upstream MEA-ATREP2 Helitron and Downstream MEA-ISR 182 bp Is Not Necessary for MEA Imprinting

\begin{tabular}{llllcccc}
\hline $\begin{array}{l}\text { Arabidopsis } \\
\text { accession }\end{array}$ & $\begin{array}{l}\text { Candidate } \\
\text { cis-acting element }\end{array}$ & $\begin{array}{l}\text { Aborted } \\
\text { F2 seeds }\end{array}$ & $\begin{array}{l}\text { Normal } \\
\text { F2 seeds }\end{array}$ & $\begin{array}{l}\text { Total } \\
\text { F2 seeds }\end{array}$ & $\begin{array}{l}\text { \% aborted } \\
\text { F2 seeds }\end{array}$ & $\chi^{2 \text { a }}$ & Best-fit model $^{\text {b }}$ \\
\hline Ler-0 & $\begin{array}{l}\text { MEA-AtREP2 and } \\
\text { MEA-ISR }(182 \mathrm{nt})_{7}\end{array}$ & 215 & 233 & 448 & 47.99 & 0.362 & no modifier \\
Yo-0 & MEA-AtREP2 absent & 1192 & 1315 & 2507 & 47.55 & 3.019 & no modifier \\
Kb-0 & MEA-AtREP2 absent & 277 & 270 & 547 & 50.64 & 0.045 & no modifier \\
Pa-1 & MEA-ISR $(182 \mathrm{nt})_{1}$ & 1274 & 1158 & 2432 & 52.38 & 2.768 & no modifier \\
\hline
\end{tabular}

aTest of contingency with the expected values for a 1:1 aborted :normal seed ratio corresponding to a "no modifier" model.

${ }^{\mathrm{b}}$ For $\alpha<0.05\left(\chi^{2}=3.84, \mathrm{df}=1\right)$. 
A

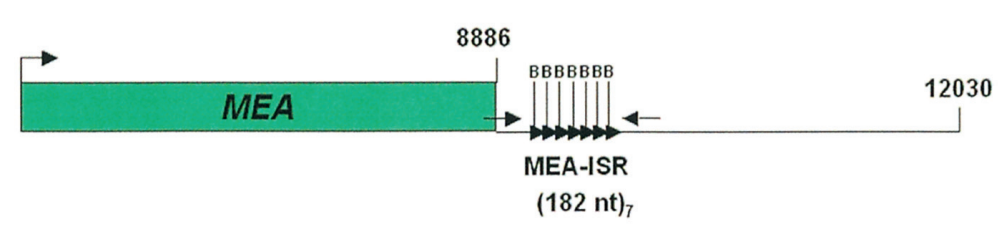

B
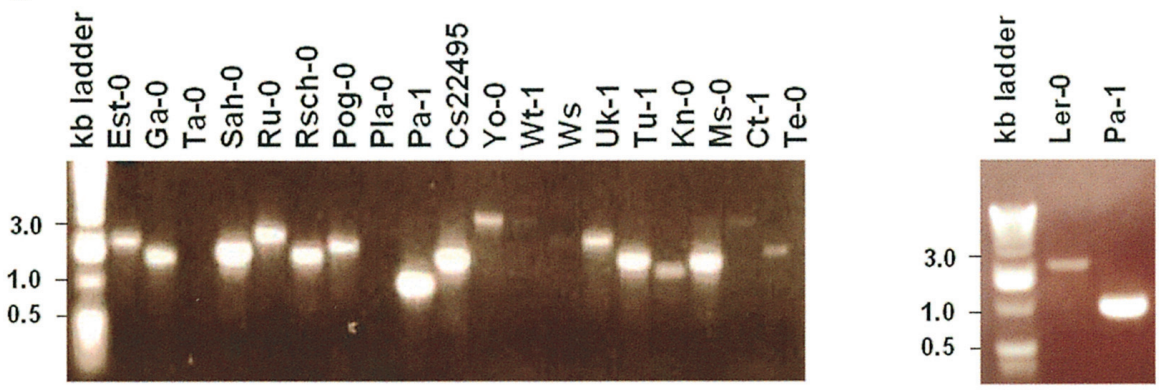

Figure 3. Expansion and contraction of the repetitive MEA-ISR tandem repeats downstream of the imprinted $M E A$ locus. $(A)$ Schematic representation of MEA-ISR region, where BamHI sites $(B)$ are contained in each 182-nucleotide repeat unit. $(B)$ Gel electrophoresis of PCR products using primers spanning the MEA-ISR region indicate that the MEA-ISR region undergoes expansions and contractions because of differing numbers of repeat units.

shown). Sequencing of the smallest PCR products allowed the identification of an ecotype (Pa-1) containing only a single MEA-ISR repeat copy (i.e., no repeats of the 182 nucleotides). As the MEA locus remains imprinted in the $\mathrm{Pa}-1$ accession, which contains no modifiers of $M E A$ imprinting (Table 1), we conclude that tandem repeats in the MEA-ISR region do not constitute an essential cisacting imprinting control region for the $M E A$ locus.

\section{Identification of a Promoter Region Sufficient for Parent-of-Origin-dependent Expression Rules Out a Role for Potential Epigenetic Attractors}

To test independently for regions required for imprinted expression of $M E A$, we investigated whether a promoter fragment driving a reporter gene can reproduce the imprinted expression pattern of $M E A$. Transgenic experiments provided evidence that $c i s$-acting elements required for imprinting are present in the proximal upstream region of the $M E A$ gene. A truncated promoter fragment missing these candidate sequences, but spanning the $\mathrm{CpG}$ islands, and comprising the first intron of the $M E A$ open reading frame (Fig. 4A), is able to confer imprinted expression on the bacterial uidA reporter gene encoding $\beta$-glucuronidase (GUS). Reciprocal crosses between transgenic Arabidopsis lines carrying the MEAp:GUS construct and wild-type plants demonstrated that this promoter fragment is maternally active but paternally inactive in the embryo and endosperm, thereby recapitulating the imprinted expression profile of the endogenous $M E A$ gene (Fig. 4B). In agreement with our natural variation studies, these results strongly suggest that the potential epigenetic attractor sites are not required for the parent-of-origin-dependent expression of $M E A$. Neither the AtREP2 helitron found in the upstream region nor the direct repeats located in 3 ' region of the $M E A$ gene (both the 187-nucleotide repeats and the GAA trinucleotide repeats) are involved in genomic imprinting at the $M E A$ locus.

\section{CONCLUSIONS}

In this report we have reviewed the potential role of transposons and repeated sequences in epigenetic gene regulation. There is accumulating evidence that such elements can serve as attractors of epigenetic regulation and that they are involved in gene silencing, paramutation, and genomic imprinting. Recently, a role for the AtSINE3 transposon in genomic imprinting at the $F W A$ locus in Arabidopsis was reported (Lippman et al. 2004). In contrast, we could not find evidence for the involvement in genomic imprinting of a transposon upstream, or direct repeat seqences (MEA-ISR and GAA trinucleotide repeats) downstream, of the $M E A$ locus. This difference suggests that distinct molecular mechanisms are involved in epigenetic gene regulation by genomic imprinting in plants.

At the FWA locus, the AtSINE3 transposon inserted close to the gene and, in fact, contributes the first two exons to the FWA gene (Lippman et al. 2004). Thus, a genetic change led to the formation of a chimeric gene consisting partly of a transposon, which in turn attracts epigenetic modifications. How the transposon is affected differentially in male versus female germ cells, as are several transposons in mammals, is an open question that will attract much attention in the future. At present, very little is known about the function of epigenetic mechanisms during gametogenesis in plants. Of the many Arabidopsis mutants affecting epigenetic processes, effects in the gametes have been reported only for mutations in MET1 (Saze et al. 2003). Our study on the MEA locus, 


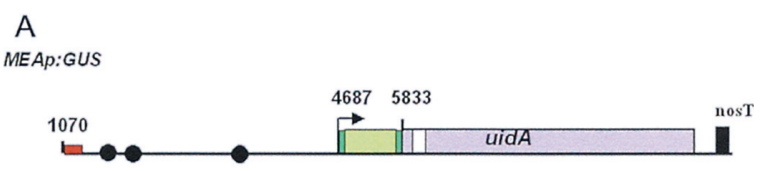

B
MEAp:GUS x wt

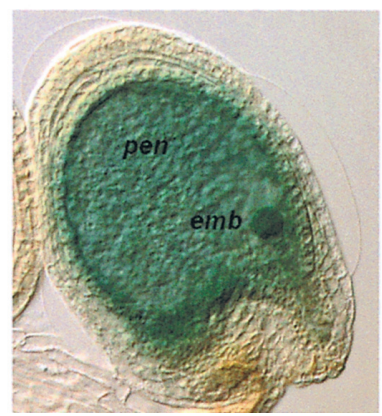

wt x MEAp:GUS

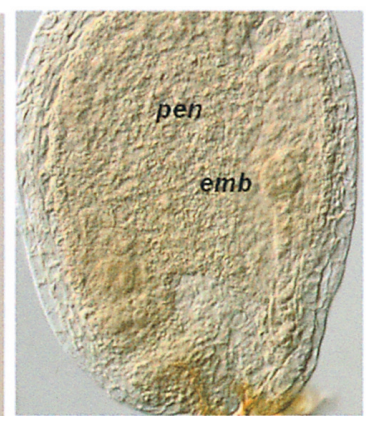

Figure 4. The AtREP2 helitron and the direct repeats are not required for imprinting. (A) The MEAp:GUS transgene comprises the uidA reporter gene (purple) under the transcriptional control of a $4.8-\mathrm{kb}$ medea genomic fragment. The fragment spans 3.8kb promoter sequences lacking the AtREP2 transposon, except for its distal $70 \mathrm{bp}$, and incorporates $1 \mathrm{~kb}$ upstream open reading frame (green; intron in pale green), but lacks the direct repeats from the 3 ' UTR. (B) Imprinted activity of the truncated promoter in developing seeds. Arabidopsis lines carrying the MEAp:GUS transgene are crossed as female (left panel) or male (right panel) to wild-type plants (wt). Transgene expression is monitored by the histochemical localization of the GUS enzyme (blue) encoded by the uidA gene. Abbreviations: pen, peripheral endosperm; emb, embryo at a globular stage.

which ruled out an essential role in imprinting for conspicuous elements such as transposons and repeats, shows that other mechanisms exist as well. The further dissection of cis-acting elements required for imprinted expression and of trans-acting factors regulating maternal and paternal $M E A$ alleles promises to shed light on these alternative mechanisms.

\section{ACKNOWLEDGMENTS}

We thank Tom Mitchell-Olds for providing us with a list of an Arabidopsis core collection that samples the most divergent accessions and was the basis for our approach relying on natural variation. Our gratitude goes to Shane McManus, who helped with the analysis of helitron variation while he was an exchange student in our lab in summer 2001 and to Andrea Steimer for helpful comments on the manuscript. This project was supported by the University of Zürich, Roche Research Foundation Fellowships to C.B. and D.R.P., as well as grant 3164061.00 of the Swiss National Science Foundation and a Searle Scholarship to U.G.

\section{REFERENCES}

Alleman M. and Doctor J. 2000. Genomic imprinting in plants: Observations and evolutionary implications. Plant Mol. Biol. 43: 147.
Arabidopsis Genome Initiative. 2000. Analysis of the genome sequence of the flowering plant Arabidopsis thaliana. Nature 408: 796.

Argeson A.C., Nelson K.K., and Siracusa L.D. 1996. Molecular basis of the pleiotropic phenotype of mice carrying the hypervariable yellow (Ahvy) mutation at the agouti locus. Genetics 142: 557 .

Bailey J.A., Carrel L., Chakravarti A., and Eichler E.E. 2000. Molecular evidence for a relationship between LINE-1 elements and $\mathrm{X}$ chromosome inactivation: The Lyon repeat hypothesis. Proc. Natl. Acad. Sci. 97: 6634.

Baroux C., Spillane C., and Grossniklaus U. 2002. Evolutionary origins of the endosperm in flowering plants. Genome Biol. 3: 1026.1.

Bender J. 2004. Chromatin-based silencing mechanisms. Curr. Opin. Plant Biol. 7: 521.

Bestor T.H. 2003. Cytosine methylation mediates sexual conflict. Trends Genet. 19: 185.

Brink R.A. 1973. Paramutation. Annu. Rev. Genet. 7: 129.

Cao X. and Jacobsen S.E. 2002. Locus-specific control of asymmetric and $\mathrm{CpNpG}$ methylation by the DRM and CMT3 methyltransferase genes. Proc. Natl. Acad. Sci. 99: 16491.

Chang-Yeh A., Mold D.E., Brilliant M.H., and Huang R.C. 1993. The mouse intracisternal A particle-promoted placental gene retrotransposition is mouse-strain-specific. Proc. Natl. Acad. Sci. 90: 292.

Chaudhuri S. and Messing J. 1994. Allele-specific parental imprinting of $d z r l$, a posttranscriptional regulator of zein accumulation. Proc. Natl. Acad. Sci. 91: 4867.

Chaudhury A.M., Ming L., Miller C., Craig S., Dennis E.S., and Peacock W.J. 1997. Fertilization-independent seed development in Arabidopsis thaliana. Proc. Natl. Acad. Sci. 94: 4223.

Choi Y., Harada J.J., Goldberg R.B., and Fischer R.L. 2004. An invariant aspartic acid in the DNA glycosylase domain of DEMETER is necessary for transcriptional activation of the imprinted MEDEA gene. Proc. Natl. Acad. Sci. 101: 7481.

Choi Y., Gehring M., Johnson L., Hannon M., Harada J.J., Goldberg R.B., Jacobsen S.E., and Fischer R.L. 2002. DEMETER, a DNA glycosylase domain protein, is required for endosperm gene imprinting and seed viability in Arabidopsis. Cell 110: 33.

Danilevskaya O.N., Hermon P., Hantke S., Muszynski M.G., Kollipara K., and Ananiev E.V. 2003. Duplicated fie genes in maize: Expression pattern and imprinting suggest distinct functions. Plant Cell 15: 425.

Dawe R.K. 2003. RNA interference, transposons, and the centromere. Plant Cell 15: 297.

Della Vedova C.B. and Cone K.C. 2004. Paramutation: The chromatin connection. Plant Cell 16: 1358.

Dorweiler J.E., Carey C.C., Kubo K.M., Hollick J.B., Kermicle J.L., and Chandler V.L. 2000. mediator of paramutation 1 is required for establishment and maintenance of paramutation at multiple maize loci. Plant Cell 12: 2101.

Edwards K., Johnstone C., and Thompson C. 1991. A simple and rapid method for the preparation of plant genomic DNA for PCR analysis. Nucleic Acids Res. 19: 1349.

Fazzari M.J. and Greally J.M. 2004. Epigenomics: Beyond CpG islands. Nat. Rev. Genet. 5: 446.

Frank M.J., Preuss D., Mack A., Kuhlmann T.C., and Crawford N.M. 1998. The Arabidopsis transposable element Tag1 is widely distributed among Arabidopsis ecotypes. Mol. Gen. Genet. 257: 478.

Gendrel A.V., Lippman Z., Yordan C., Colot V., and Martienssen R.A. 2002. Dependence of heterochromatic histone H3 methylation patterns on the Arabidopsis gene DDM1. Science 297: 1871.

Greally J.M. 2002. Short interspersed transposable elements (SINEs) are excluded from imprinted regions in the human genome. Proc. Natl. Acad. Sci. 99: 327.

Grossniklaus U., Spillane C., Page D.R., and Köhler C. 2001. Genomic imprinting and seed development: Endosperm formation with and without sex. Curr. Opin. Plant Biol. 4: 21.

Grossniklaus U., Vielle-Calzada J.P., Hoeppner M.A., and Gagliano W.B. 1998. Maternal control of embryogenesis by 
MEDEA, a Polycomb group gene in Arabidopsis. Science 280: 446

Guitton A.-E., Page D.R., Chambrier P., Lionnet C., Faure J.-E., Grossniklaus U., and Berger F. 2004. Identification of new members of Fertilisation Independent Seed Polycomb Group pathway involved in the control of seed development in Arabidopsis thaliana. Development 131: 2971.

Guo M., Rupe M.A., Danilevskaya O.N., Yang X., and Hu Z. 2003. Genome-wide mRNA profiling reveals heterochronic allelic variation and a new imprinted gene in hybrid maize endosperm. Plant J. 36: 30.

Gutiérrez-Marcos J.F., Costa L.M., Biderre-Petit C., Khbaya B., O'Sullivan D.M., Wormald M., Perez P., and Dickinson H.G. 2004. Maternally expressed gene1 is a novel maize endosperm transfer cell-specific gene with a maternal parent-oforigin pattern of expression. Plant Cell 16: 1288.

Hirochika H., Okamoto H., and Kakutani T. 2000. Silencing of retrotransposons in Arabidopsis and reactivation by the $d d m 1$ mutation. Plant Cell 12: 357.

Imhof A. 2003. Histone modifications: An assembly line for active chromatin? Curr. Biol. 13: R22.

Kapitonov V.V. and Jurka J. 2001. Rolling-circle transposons in eukaryotes. Proc. Natl. Acad. Sci. 98: 8714.

Kato M., Miura A., Bender J., Jacobsen S.E., and Kakutani T. 2003. Role of CG and non-CG methylation in immobilization of transposons in Arabidopsis. Curr. Biol. 13: 421.

Kazazian H.H., Jr. 2004. Mobile elements: Drivers of genome evolution. Science 303: 1626.

Kermicle J.L. 1970. Dependence of the $R$-mottled aleurone phenotype in maize on mode of sexual transmission. Genetics 66: 69.

1994. Epigenetic silencing and activation of a maize $r$ gene. In Epigenetic mechanisms of gene regulation (ed. V.E.A. Russo et al.), p. 267. Cold Spring Harbor Laboratory Press, Cold Spring Harbor, New York.

Kinoshita T., Yadegari R., Harada J.J., Goldberg R.B., and Fischer R.L. 1999. Imprinting of the MEDEA Polycomb gene in the Arabidopsis endosperm. Plant Cell 11: 1945.

Kinoshita T., Miura A., Choi Y., Kinoshita Y., Cao X., Jacobsen S.E., Fischer R.L., and Kakutani T. 2004. One-way control of FWA imprinting in Arabidopsis endosperm by DNA methylation. Science 303: 521.

Köhler C., Hennig L., Bouveret R., Gheyselinck J., Grossniklaus U., and Gruissem W. 2003. Arabidopsis MSI1 is a component of the MEA/FIE Polycomb group complex and required for seed development. EMBO J. 22: 4804.

Koornneef M., Hanhart C.J., and van der Veen J.H. 1991. A genetic and physiological analysis of late flowering mutants in Arabidopsis thaliana. Mol. Gen. Genet. 229: 57.

Lewis A., Mitsuya K., Constancia M., and Reik W. 2004. Tandem repeat hypothesis in imprinting: Deletion of a conserved direct repeat element upstream of $\mathrm{H} 19$ has no effect on imprinting in the Igf2-H19 region. Mol. Cell. Biol. 24: 5650.

Lippman Z. and Martienssen R. 2004. The role of RNA interference in heterochromatin silencing. Nature 431: 364.

Lippman Z., May B., Yordan C., Singer T., and Martienssen R. 2003. Distinct mechanisms determine transposon inheritance and methylation via small interfering RNA and histone modification. PLoS Biol. 1: E67.

Lippman Z., Gendrel A.V., Black M., Vaughn M.W., Dedhia N., McCombie W.R., Lavine K., Mittal V., May B., Kasschau K.D., Carrington J.C., Doerge R.W., Colot V., and Martienssen R. 2004. Role of transposable elements in heterochromatin and epigenetic control. Nature 430: 471.

Lisch D., Carey C.C., Dorweiler J.E., and Chandler V.L. 2002. A mutation that prevents paramutation in maize also reverses Mutator transposon methylation and silencing. Proc. Natl. Acad. Sci. 99: 6130.

Lund G., Ciceri P., and Viotti A. 1995a. Maternal-specific demethylation and expression of specific alleles of zein genes in the endosperm of Zea mays L. Plant J. 8: 571.

Lund G., Messing J., and Viotti A. 1995b. Endosperm-specific demethylation and activation of specific alleles of alpha- tubulin genes of Zea mays L. Mol. Gen. Genet. 246: 716

Luo M., Bilodeau P., Dennis E.S., Peacock W.J., and Chaudhury A. 2000. Expression and parent-of-origin effects for FIS2, $M E A$, and FIE in the endosperm and embryo of developing Arabidopsis seeds. Proc. Natl. Acad. Sci. 97: 10637.

Luo M., Bilodeau P., Koltunow A., Dennis E.S., Peacock W.J., and Chaudhury A.M. 1999. Genes controlling fertilization-independent seed development in Arabidopsis thaliana. Proc. Natl. Acad. Sci. 96: 296.

Lyon M.F. 2003. The Lyon and the LINE hypothesis. Semin. Cell Dev. Biol. 14: 313.

Martienssen R.A. 1996. Epigenetic phenomena: Paramutation and gene silencing in plants. Curr. Biol. 6: 810.

Martienssen R.A. and Colot V. 2001. DNA methylation and epigenetic inheritance in plants and filamentous fungi. Science 293: 1070.

Matzke M.A., Matzke A.J.M., and Eggleston W.B. 1996. Paramutation and transgene silencing: A common response to invasive DNA? Trends Plant Sci. 1: 382.

McClintock B. 1952. Chromosome organization and genic expression. Cold Spring Harbor Symp. Quant. Biol. 16: 13.

. 1984. The significance of responses of the genome to challenge (Nobel Lecture). Science 226: 792.

Michaud E.J., van Vugt M.J., Bultman S.J., Sweet H.O., Davisson M.T., and Woychik R.P. 1994. Differential expression of a new dominant agouti allele (Aiapy) is correlated with methylation state and is influenced by parental lineage. Genes Dev. 8: 1463.

Moore T. 2001. Genetic conflict, genomic imprinting and establishment of the epigenotype in relation to growth. Reproduction 122: 185 .

Morgan H.D., Sutherland H.G., Martin D.I., and Whitelaw E. 1999. Epigenetic inheritance at the agouti locus in the mouse. Nat. Genet. 23: 314.

Neumann B., Kubicka P., and Barlow D.P. 1995. Characteristics of imprinted genes. Nat. Genet. 9: 12.

Ohad N., Margossian L., Hsu Y.C., Williams C., Repetti P., and Fischer R.L. 1996. A mutation that allows endosperm development without fertilization. Proc. Natl. Acad. Sci. 93: 5319.

Ohad N., Yadegari R., Margossian L., Hannon M., Michaeli D., Harada J.J., Goldberg R.B., and Fischer R.L. 1999. Mutations in FIE, a WD polycomb group gene, allow endosperm development without fertilization. Plant Cell 11: 407.

Page D.R. 2004. "Maternal effects during seed development in Arabidopsis thaliana: An expression analysis of the FIS class gene MEDEA and a search for new FIS class mutants." Ph.D. thesis, University of Zürich, Zürich, Switzerland.

Rakyan V.K., Blewitt M.E., Druker R., Preis J.I., and Whitelaw E. 2002. Metastable epialleles in mammals. Trends Genet. 18: 348.

Rakyan V.K., Chong S., Champ M.E., Cuthbert P.C., Morgan H.D., Luu K.V., and Whitelaw E. 2003. Transgenerational inheritance of epigenetic states at the murine Axin( $\mathrm{Fu}$ ) allele occurs after maternal and paternal transmission. Proc. Natl. Acad. Sci. 100: 2538.

Rea S., Eisenhaber F., O'Carroll D., Strahl B.D., Sun Z.W., Schmid M., Opravil S., Mechtler K., Ponting C.P., Allis C.D., and Jenuwein T. 2000. Regulation of chromatin structure by site-specific histone H3 methyltransferases. Nature 406: 593.

Reyes J.C. and Grossniklaus U. 2003. Diverse functions of Polycomb group proteins during plant development. Semin. Cell Dev. Biol. 14: 77.

Rubin C.M., VandeVoort C.A., Teplitz R.L., and Schmid C.W. 1994. Alu repeated DNAs are differentially methylated in primate germ cells. Nucleic Acids Res. 22: 5121.

Sanford J.P., Clark H.J., Chapman V.M., and Rossant J. 1987. Differences in DNA methylation during oogenesis and spermatogenesis and their persistence during early embryogenesis in the mouse. Genes Dev. 1: 1039.

Saze H., Mittelsten Scheid O., and Paszkowski J. 2003. Maintenance of $\mathrm{CpG}$ methylation is essential for epigenetic inheritance during plant gametogenesis. Nat. Genet. 34: 65.

Soppe W.J., Jacobsen S.E., Alonso-Blanco C., Jackson J.P., 
Kakutani T., Koornneef M., and Peeters A.J. 2000. The late flowering phenotype of fwa mutants is caused by gain-offunction epigenetic alleles of a homeodomain gene. Mol. Cell 6: 791 .

Spillane C., MacDougall C., Stock C., Kohler C., Vielle-Calzada J.P., Nunes S.M., Grossniklaus U., and Goodrich J. 2000. Interaction of the Arabidopsis Polycomb group proteins FIE and MEA mediates their common phenotypes. Curr. Biol. 10: 1535.

Springer N.M., Danilevskaya O.N., Hermon P., Helentjaris T.G., Phillips R.L., Kaeppler H.F., and Kaeppler S.M. 2002. Sequence relationships, conserved domains, and expression patterns for maize homologs of the Polycomb group genes $E(z), e s c$, and $E(P c)$. Plant Physiol. 128: 1332.

Stam M., Belele C., Dorweiler J.E., and Chandler V.L. 2002. Differential chromatin structure within a tandem array $100 \mathrm{~kb}$ upstream of the maize $b 1$ locus is associated with paramutation. Genes Dev. 16: 1906.

Tabara H., Sarkissian M., Kelly W.G., Fleenor J., Grishok A., Timmons L., Fire A., and Mello C.C. 1999. The rde-1 gene, RNA interference, and transposon silencing in C. elegans. Cell 99: 123.

Tariq M. and Paszkowski J. 2004. DNA and histone methylation in plants. Trends Genet. 20: 244.

van de Lagemaat L.N., Landry J.R., Mager D.L., and Medstrand P. 2003. Transposable elements in mammals promote regulatory variation and diversification of genes with specialized functions. Trends Genet. 19: 530.

Vielle-Calzada J.P., Thomas J., Spillane C., Coluccio A., Hoeppner M.A., and Grossniklaus U. 1999. Maintenance of genomic imprinting at the Arabidopsis medea locus requires zygotic DDM1 activity. Genes Dev. 13: 2971.

Volpe T.A., Kidner C., Hall I.M., Teng G., Grewal S.I., and Martienssen R.A. 2002. Regulation of heterochromatic silencing and histone H3 lysine-9 methylation by RNAi. Science 297: 1833 .

Walbot V. 2001. Imprinting of $R-r$, paramutation of $B-I$ and $P l$, and epigenetic silencing of $M u D R / M u$ transposons in $Z e a$ mays L. are coordinately affected by inbred background. Genet. Res. 77: 219.

Walker E.L. and Panavas T. 2001. Structural features and methylation patterns associated with paramutation at the $r l$ locus of Zea mays. Genetics 159: 1201.

Walsh C.P., Chaillet J.R., and Bestor T.H. 1998. Transcription of IAP endogenous retroviruses is constrained by cytosine methylation. Nat. Genet. 20: 116.

Wang Y., Fischle W., Cheung W., Jacobs S., Khorasanizadeh S., and Allis C.D. 2004. Beyond the double helix: Writing and reading the histone code. Novartis Found. Symp. 259: 3.

Whitelaw E. and Martin D.I. 2001. Retrotransposons as epigenetic mediators of phenotypic variation in mammals. Nat. Genet. 27: 361

Xiao W., Gehring M., Choi Y., Margossian L., Pu H., Harada J.J., Goldberg R.B., Pennell R.I., and Fischer R.L. Imprinting of the MEA Polycomb gene is controlled by antagonism between MET1 methyltransferase and DME glycosylase. Dev. Cell 5: 891 .

Yadegari R., Kinoshita T., Lotan O., Cohen G., Katz A., Choi Y., Nakashima K., Harada J.J., Goldberg R.B., Fischer R.L., and Ohad N. 2000. Mutations in the FIE and MEA genes that encode interacting Polycomb proteins cause parent-of-origin effects on seed development by distinct mechanisms. Plant Cell 12: 2367.

Yates P.A., Burman R.W., Mummaneni P., Krussel S., and Turker M.S. 1999. Tandem B1 elements located in a mouse methylation center provide a target for de novo DNA methylation. J. Biol. Chem. 274: 36357.

Yoder J.A., Walsh C.P., and Bestor T.H. 1997. Cytosine methylation and the ecology of intragenomic parasites. Trends Genet. 13: 335 .

Zilberman D., Cao X., and Jacobsen S.E. 2003. ARGONAUTE4 control of locus-specific siRNA accumulation and DNA and histone methylation. Science 299: 716 . 


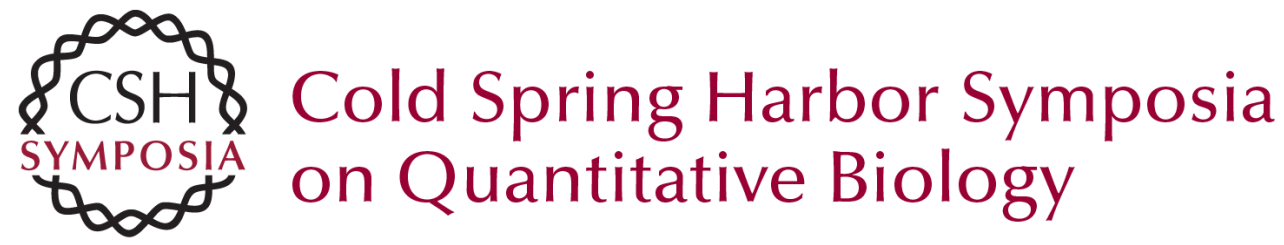

\section{Transposons and Tandem Repeats Are Not Involved in the Control of Genomic Imprinting at the MEDEA Locus in Arabidopsis}

C. SPILLANE, C. BAROUX, J.-M. ESCOBAR-RESTREPO, et al.

Cold Spring Harb Symp Quant Biol 2004 69: 465-476

Access the most recent version at doi:10.1101/sqb.2004.69.465

References This article cites 84 articles, 40 of which can be accessed free at: http://symposium.cshlp.org/content/69/465.full.html\#ref-list-1

\section{License}

Email Alerting Receive free email alerts when new articles cite this article - sign up in Service the box at the top right corner of the article or click here. 